\title{
Pour une génétique de l'improvisation musicale (première partie)
}

Éléments théoriques

\section{Clément Canonne et Martin Guerpin}

\section{Q OpenEdition}

\section{Journals}

\section{Édition électronique}

URL : http://journals.openedition.org/genesis/3749

DOI : $10.4000 /$ genesis.3749

ISSN : 2268-1590

\section{Éditeur :}

Presses universitaires de Paris Sorbonne (PUPS), Société internationale de génétique artistique littéraire et scientifique (SIGALES)

\section{Édition imprimée}

Date de publication : 15 décembre 2018

Pagination : 155-167

ISBN : 979-10-231-06282

ISSN : 1167-5101

\section{Référence électronique}

Clément Canonne et Martin Guerpin, «Pour une génétique de l'improvisation musicale (première partie) », Genesis [En ligne], 47 | 2018, mis en ligne le 15 décembre 2019, consulté le 21 janvier 2021. URL : http://journals.openedition.org/genesis/3749; DOI : https://doi.org/10.4000/genesis.3749 


\section{Pour une génétique de l'improvisation musicale Première partie : éléments théoriques}

\section{Clément Canonne et Martin Guerpin}

$\mathrm{C}$ ela fait bien longtemps que le Génie (solitaire) et l'Inspiration (fulgurante) ont été chassés du paradis des créateurs. Toute une série de travaux ont contribué à relativiser ces mythes en dissipant une partie des nimbes qui entouraient la création artistique 1 . À ce mouvement de désacralisation, la génétique littéraire a apporté son écot. C'est toutefois dans le domaine de l'improvisation que la dimension mystérieuse de la création trouve aujourd'hui l'un de ses derniers refuges : l'improvisation reste fortement associée à l'idée d'une création sur le vif, directe et spontanée. En effet, s'il est vrai que, depuis les années 1990, les études sur les processus créatifs à l'œuvre dans l'improvisation ont suscité un nombre croissant de travaux en musicologie, en ethnomusicologie, en philosophie, en psychologie cognitive ou encore en anthropologie, la tendance générale reste soit de considérer la performance improvisée pour elle-même (par exemple en s'intéressant aux interactions in situ entre les musiciens ${ }^{2}$ ), soit de la replacer dans le cadre de pratiques beaucoup plus larges qui ont essentiellement à voir avec la formation des improvisateurs ${ }^{3}$ ou avec l'environnement culturel et esthétique dans lequel ils évoluent ${ }^{4}$. Mais dans tous les cas, ce sont les «avants» les plus immédiats de l'improvisation qui se trouvent négligés.

L'ambition de cet article consiste donc à proposer, en s'inspirant de la génétique littéraire, une nouvelle approche des processus de création à l'œuvre dans l'improvisation. Nous les envisagerons à partir de deux genres musicaux recouvrant une grande variété de pratiques improvisées : le jazz et les musiques dites d'improvisation libre5. Quelles sont les conditions de possibilité d'une génétique de l'improvisation musicale? Sur quoi porte-t-elle exactement? Quels types de processus permet-elle de mettre au jour? Et quels sont les apports potentiels d'une approche génétique à la compréhension des pratiques improvisées mais aussi, plus largement, de l'invention musicale? En raison de l'absence de travaux explicitement consacrés à ces questions, notre entreprise revêt une dimension expérimentale, prospective et programmatique. Expérimentale, car les réflexions proposées sont issues de questions soulevées par une série de travaux que nous avons initiée à partir de cas d'études particuliers. Prospective, parce que l'article s'attache à définir une direction et une approche nouvelles dans le domaine de l'improvisation musicale et à anticiper de possibles travaux à venir. Programmatique, enfin, parce nous délimitons les cadres au sein desquels pourront s'inscrire de futures études de cas.

L'élaboration d'une approche génétique de l'improvisation met en jeu un nouvel élargissement des études génétiques. Une première extension de leur domaine a été réalisée au moment du transfert des approches génétiques de la littérature à la composition musicale. Ce transfert a provoqué un renouvellement significatif des études des esquisses et des brouillons musicaux. Encouragées par la série de conférences «Analyser les processus de création musicale» (Lille 2011, Montréal 2013, Paris 2015, Huddersfield 2017), les travaux consacrés aux processus à l'œuvre dans les pratiques compositionnelles sont aujourd'hui devenus monnaie courante. La revue Genesis a d'ailleurs joué un rôle fondateur dans

1. Voir par exemple Jack Stillinger, Multiple Authorship and the Myth of Solitary Genius, New York, Oxford University Press, 2011.

2. Ingrid Monson, Saying Something. Jazz Improvisation and Interaction, Chicago, Chicago University Press, coll. "Chicago Studies in Ethnomusicology », 1996; Keith Sawyer, «Group Creativity : Musical Performance and Collaboration», Psychology of Music, vol. 34, n 2, 2006, p. 148-165; «Improvisation collective libre et processus de création musicale : création et créativité au prisme de la coordination», Revue de Musicologie, vol. 98, $\mathrm{n}^{\circ}$ 1, p. 107-148.

3. Paul Berliner, Thinking in Jazz. The Infinite Art of Improvisation, Chicago, Chicago University Press, coll. "Chicago Studies in Ethnomusicology», 1994.

4. Matthieu Saladin, Esthétique de l'improvisation libre. Expérimentation musicale et politique, Dijon, Les Presses du Réel, 2014.

5. L'improvisation libre désigne un ensemble de pratiques issues $\mathrm{du}$ « free jazz des années 1960-1970 et [de] cette esthétique de l'indétermination que rencontre une certaine musique contemporaine dans les années 19501960 » (Clément Canonne, «Improvisation collective libre et processus de création musicale», 2012, p. 115). Voir également John Corbett, A Listener's Guide to Free Improvisation, Chicago, University of Chicago Press, 2016. 
cette première extension du domaine de la critique génétique, puisque, dès 1993, la composition musicale y a occupé une place importante 6 . Rétrospectivement, cet élargissement peut paraître tout naturel puisque les processus de création envisagés dans le domaine de la littérature et de la musique ont pour point commun de mettre en jeu l'écriture, qui sert de passerelle entre les deux domaines.

Or, nous faisons l'hypothèse que les approches génétiques peuvent faire l'objet d'une seconde extension et s'appliquer à des processus de création dans lesquels le rôle de l'écriture est marginal, voire inexistant. L'examen de cette hypothèse est au cœur de la réflexion proposée ici. D'emblée, elle pose au regard de la critique génétique un problème ontologique et méthodologique. D'une part, en effet, le mode d'être de la musique improvisée se distingue radicalement de celui de la musique notée. D'autre part, l'improvisation ne laisse pas (ou très peu, et de manière non décisive) ces traces écrites qui constituent les matériaux privilégiés des approches génétiques. Fonder une génétique de l'improvisation musicale ne va donc pas de soi.

Face à un tel constat, il paraît nécessaire de passer en revue les différents problèmes conceptuels que pose le statut ontologique de l'improvisation à une approche génétique qui lui serait consacrée. Nous introduirons ensuite la notion de «projet improvisatoire» et montrerons de quelle façon l'existence de tels projets conditionne la possibilité d'une approche génétique de l'improvisation. Dans un troisième temps (voir la seconde partie de l'article dans le prochain numéro de la revue Genesis), nous questionnerons d'un point de vue théorique la possibilité de transférer à la génétique de l'improvisation les outils et les méthodes propres à la critique génétique textuelle. Enfin, une fois le cadre théorique et méthodologique de la génétique de l'improvisation fixé, nous suggérerons une typologie d'applications possibles, illustrées par des cas d'étude.

\section{Les conditions de possibilité d'une génétique de l'improvisation musicale}

La génétique de l'improvisation ne peut être conçue comme une simple application de la critique génétique à un objet nouveau. Afin de préserver les apports caractéristiques de cette approche et de ne pas en proposer un équivalent vague ou mal fondé, plusieurs difficultés doivent être prises en compte.

\section{Le problème de l'évanescence de l'improvisation}

La première difficulté réside dans l'évanescence de l'improvisation. Comme toute performance musicale, une improvisation est constituée d'une suite d'événements sonores au premier chef, mais également visuels et tactiles qui apparaissent puis s'évanouissent sans laisser aucune trace (sinon mémorielle). Dans les termes de Lee B. Brown (2014), l'improvisation n'est pas un “continuant", c'est-à-dire qu'elle n'est pas réidentifiable au cours du temps comme peuvent l'être par exemple la Symphonie pastorale de Beethoven ou le Guernica de Picasso ${ }^{7}$. Or il ne peut y avoir de génétique sans traces matérielles endurantes - susceptibles à la fois d'être mises à distance, en vue d'une analyse critique, et d'être partagées avec d'autres, dans l'administration de la preuve. La génétique de l'improvisation que nous visons ici se ramène donc nécessairement à une génétique de l'improvisation enregistrée - quelle que soit par ailleurs la nature des enregistrements étudiés (audio ou vidéo; autorisé par le musicien ou non; réalisé à des fins de documentation ethnographique ou de diffusion commerciale, etc. $)^{8}$. En d'autres termes, la présence de traces sonores enregistrées constitue la condition de possibilité matérielle d'une génétique de l'improvisation.

6. Nous faisons référence au numéro pionnier intitulé «Écritures musicales aujourd'hui» coordonné par Peter Szendy (Genesis, n 4, 1993), ainsi qu'aux numéros intitulés «Composer» coordonné par Nicolas Donin (Genesis, $\mathrm{n}^{\circ} 31,2010$ ) et «Créer à plusieurs mains » coordonné par Nicolas Donin et Daniel Ferrer (Genesis, ${ }^{\circ}$ 41, 2015). Voir aussi William Kindermann, «Les étapes de la genèse de la Sonate en mi majeur op. 109 de Beethoven», Genesis, n² 29, 2008, p. 9-28.

7. Voir Lee B. Brown, «Euvres de musique, improvisation et principe de continuité», dans Alessandro Arbo et Marcello Ruta (dir.), L'Ontologie musicale : perspectives et débat, Paris, Hermann, 2014, p. 235-278.

8. Cette condition n'a rien d'insurmontable, tant les musiques improvisées, et le jazz en particulier, dans leur pratique comme dans leur histoire, sont fondamentalement liées à l'enregistrement. Comme le rappelle Laurent Cugny à propos du jazz, «la phonographie a façonné tout le processus de transmission (apprentissage, diffusion, commercialisation...) [du jazz]. C'est par les disques qu'on découvre le plus souvent cette musique, qu'on l'apprend, qu' on la fait circuler, qu'on la retient, qu'on l'étudie» (Laurent Cugny, Analyser le Jazz, Paris, Outre Mesure, 2009 , p. 71). Pour un examen plus général de la relation entre improvisation et enregistrement, et une réponse aux arguments défendant la thèse d'une incompatibilité entre improvisation et enregistrement, voir Clément Canonne, «Improvisation et enregistrement», dans Alessandro Arbo et Pierre-Emmanuel Lephay (dir.), Quand l'enregistrement change la musique, Paris, Hermann, 2017, p. 195-217. 


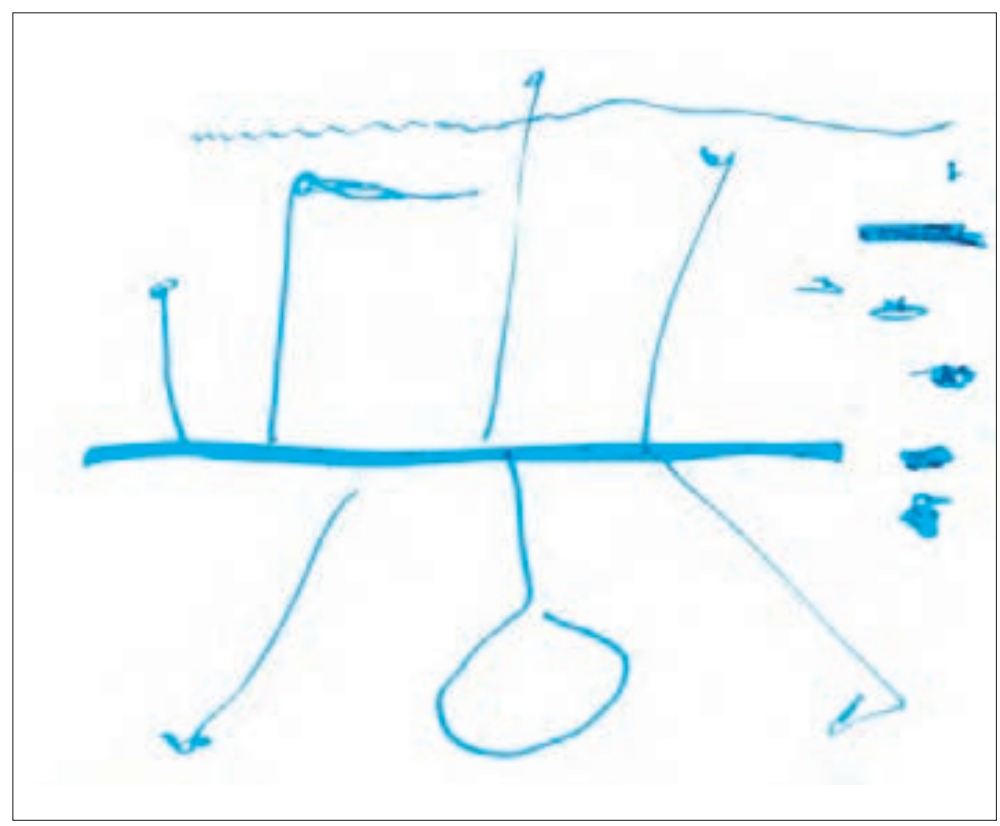

Fig. 1a : Dessin de la pianiste Ève Risser qui propose une description graphique de la musique improvisée du duo Risser-Rühl.

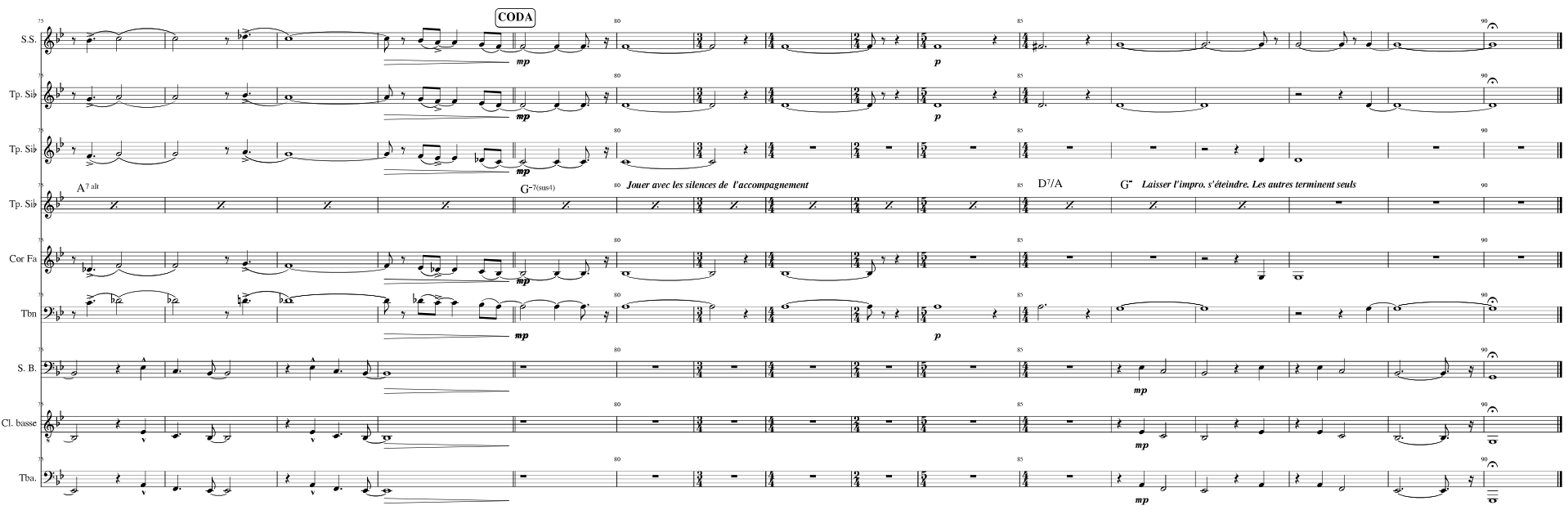

Fig. 1b : Grille d'improvisation de Pesantes heures en apesanteur (morceau composé par Martin Guerpin pour le Gil Evans Paris Workshop Horns Only, 2018), mes. 75-90.

Certes, on pourrait également tenter de retracer la genèse d'une improvisation donnée à partir des documents écrits laissés par les improvisateurs - notes manuscrites, dessins, schémas, partitions, etc. Mais si ces documents ont assurément toute leur place au sein d'un dossier génétique, leur rôle explicatif ne saurait rivaliser avec celui des enregistrements sonores, et ce pour trois raisons :

- ces traces écrites sont rarement très nombreuses;

- quand elles existent, elles portent davantage sur le cadre compositionnel auquel s'articule la performance improvisée que sur l'improvisation elle-même (voir les figures 1a et 1b);
- sans traces sonores complémentaires, il y a un saut qualitatif inéliminable entre ces documents écrits et la musique à laquelle ils sont censés faire référence 9 .

9. Il s'agit bien sûr là d'un argument général, la notation musicale ne pouvant jamais prétendre capturer dans tous ses détails le phénomène sonore auquel elle renvoie. Mais il vaut tout particulièrement dans le cas d'une génétique de l'improvisation musicale puisque, d'une part, les potentielles traces écrites circulant dans le monde des musiques improvisées sont en général bien plus sous-déterminées (parce que plus lacunaires, plus vagues, plus elliptiques, etc.) que les partitions de la musique occidentale savante et, d'autre part, parce que le projet d'une génétique 


\section{Le problème de l'irréductible singularité de l'improvisation}

La deuxième difficulté réside dans la singularité de l'improvisation, qui est à la fois littéralement non planifiée et non répétable. À cela s'ajoute l'hyper-dépendance contextuelle de l'improvisation. Certes, à un certain degré, toute performance musicale est dépendante de son contexte. Mais à la différence de l'interprète qui doit suivre un script d'action établi en amont de la performance (la partition), l'improvisateur est libre de modeler la conduite de sa performance à partir des ressources que lui fournit son environnement 10 . Le déroulé exact d'une improvisation peut donc dépendre dans une large mesure de conditions de productions (salle, instruments, public, cadre institutionnel, etc.) qui, par définition, ne se répètent jamais à l'identique ${ }^{11}$. Or, si chaque improvisation se présente comme une performance-monade sans passé ni avenir, c'est le projet même d'une génétique de l'improvisation qui semble s'effondrer. Ou, pour le dire autrement, si l'improvisateur se donne bel et bien comme horizon l'invention sur le vif et la non-répétition d'une performance à l'autre - chaque improvisation étant alors considérée comme un nouveau commencement - on ne voit pas vraiment à quoi pourrait correspondre une génétique de l'improvisation. Cependant, s'il ne faut pas sous-estimer le rôle régulateur que peut jouer un tel idéal dans la pratique de certains improvisateurs (on peut penser au saxophoniste Lee Konitz ou au guitariste Derek Bailey), il serait quelque peu naif de considérer qu'il offre une description adéquate des performances improvisées observables au sein du jazz et des musiques improvisées.

D'une part, cette vision singulariste de l'improvisation néglige le rôle joué par le souvenir (voire la mémorisation) des performances passées dans la conduite d'une improvisation donnée. L'improvisateur improvise à partir de savoir-faire patiemment incorporés et, au moins dans le cas du jazz, à partir d'une structure préexistante (par exemple la grille harmonique d'un standard comme My Funny Valentine) qui vient guider la conduite de l'improvisation, mais il improvise aussi inévitablement à partir d'une mémoire à plus court terme ${ }^{12}$; et au sein de cette mémoire, le souvenir des improvisations passées occupe une place prépondérante, ne serait-ce que pour s'en démarquer ou pour tenter, cette fois-ci, d'explorer un autre chemin. L'influence des performances passées peut être largement inconsciente, simplement due aux traces plus ou moins définies qu'elles ont laissées dans la mémoire de l'improvisateur. Mais elle peut aussi être beaucoup plus consciente, lorsque le souvenir de ces performances passées est réactivé, que ce soit par la réécoute critique d'une prise enregistrée ou par une discussion collective au sujet d'une des performances passées du groupe. L'existence de tels processus de réécoute et de discussions critiques est relativement bien documentée dans le cas du jazz ${ }^{13}$ comme dans

de l'improvisation consiste à faire une génétique des performances musicales et non des supports matériels les encodant - comme c'est habituellement le cas des sketch studies (voir Friedemann Sallis, Music Sketches, Cambridge, Cambridge University Press, 2015). 10. Voir par exemple Jocelyn Bonnerave, «Improviser ensemble. De l'interaction à l'écologie sonore», Tracés, n 18, 2010, p. 87-103.

11. Le court solo improvisé par le pianiste Orrin Evans lors de la soirée d'ouverture de l'édition 2018 du festival Météo à Mulhouse permet d'illustrer cette hyper-dépendance contextuelle de l'improvisation. Cette improvisation a été précédée, en première partie, par un concert solo du pianiste Pat Thomas au cours duquel celui-ci a multiplié clusters et autres agrégats fortissimo dans tous les registres de l'instrument, conduisant ainsi le piano à se désaccorder fortement. Quand vient le tour d'Orrin Evans, celui-ci commence son improvisation dans un style plus traditionnellement jazz; mais au détour d'une phrase, il attaque une note particulièrement désaccordée. Il s'y arrête aussitôt, la répétant au sein d'interventions de plus en plus véhémentes - référence probable (et clairement ironique) au solo précédent de Pat Thomas. Mais l'improvisation d'Orrin Evans aurait sans doute pris un tout autre tour si les conditions du concert avaient été différentes - en l'occurrence, si l'improvisation n'avait pas été précédée par la première partie de Pat Thomas ou si le piano avait été réaccordé.

12. Voir notamment Jeff Pressing, «Psychological constraints on improvisational expertise», dans Bruno Nettl et Melinda Russell (dir.), In the Course of Performance. Studies in the World of Musical Improvisation, Chicago, University of Chicago Press, 1998, p. 47-67, pour une discussion du rôle de la mémoire dans les processus cognitifs de l'improvisateur. 13. Ainsi Miles Davis décrit-il dans son autobiographie le comportement des membres du deuxième quintette en tournée : «Tous les soirs, Herbie, Tony et Ron se retrouvaient dans leur chambre d'hôtel, et parlaient de ce qu'ils avaient joué jusqu'au petit matin. Tous les soirs, ils revenaient sur scène et jouaient quelque chose de différent. Et tous les soirs, il fallait que je réagisse. La musique qu' on jouait ensemble changeait tous les soirs » (Miles Davis et Quincy Troupe, Miles. The Autobiography, New York, Simon \& Schuster, 1989, p. 278). Comme on le voit, s'il faut en croire Miles Davis, le fait que les performances passées sont bien présentes à l'esprit des musiciens quand vient le moment du concert n'est donc nullement incompatible avec le fait que les musiciens tentent de renouveler la conduite de leurs improvisations (au moins sous certains aspects) à chaque performance. 
le cas des musiques librement improvisées ${ }^{14}$. Dans cette perspective, il est donc tout à fait légitime de s'intéresser à la manière dont les musiciens peuvent remobiliser au cours d'une improvisation donnée certains éléments issus de performances passées.

D'autre part, la vision singulariste de l'improvisation néglige toutes les possibilités qu'ont les musiciens d'anticiper une performance, en s'y préparant d'une manière ou d'une autre. Ces anticipations peuvent prendre diverses formes, des solos «composés» de Louis Armstrong15 aux séances de travail qu'organisent parfois les improvisateurs libres en vue d'un concert ${ }^{16}$, en passant par différentes formes de préparation ${ }^{17}$, mais aussi par les «chauffes » et autres «balances » qui peuvent précéder un concert ou une session d'enregistrement. Bref, il semble ici essentiel de relativiser l'idée que les improvisateurs seraient figés dans un éternel présent, se refusant de prendre en compte leurs performances passées ou de se projeter dans leurs performances à venir.

\section{Le problème de l'absence de terminus ad quem : l'improvisation comme work-in-progress}

Mais une troisième difficulté surgit alors, symétrique de la précédente. Si les performances se trouvent ainsi connectées les unes aux autres, chaque improvisation d'un musicien prenant appui (en un sens très large) sur l'improvisation qui la précède, il convient alors peut-être de concevoir le travail de l'improvisateur comme un perpétuel work-in-progress, chaque improvisation venant nourrir la suivante dans un processus de réélaboration permanent qui ne laisse aucune place à un quelconque terminus ad quem. Cette idée a été explicitement défendue par l'historien du jazz Gunther Schuller : «Les improvisations des jazzmen constituent un work-in-progress ${ }^{18}$.» Il en découle deux corollaires, introduits par Ekkehard Jost dans son livre Free Jazz ${ }^{19}$ : d'une part, «les improvisations d'un musicien forment une chaîne de phénomènes non définitifs 20 ». Or, s'il n'y a rien de «définitif», on ne voit pas très bien de quoi on pourrait faire la genèse. D'autre part, l'étude d'une improvisation implique que «l'analyste prenne en compte tout ce qu'il a pu apprendre des autres improvisations du même musicien 21 ». Et dans ce cas, même à supposer que l'on puisse identifier ce que l'on appellera des points remarquables au sein de ce work-in-progress permanent, le projet même d'une génétique de l'improvisation finirait par se dissoudre dans l'analyse de l'intégralité du corpus d'un improvisateur.

14. Voir Clément Canonne, «Rehearsing Free Improvisation? An Ethnographic Study of Free Improvisers at Work», Music Theory Online (à paraître) : à partir d'une enquête ethnographique auprès de trois groupes d'improvisation libre, cet article montre à quel point discussions collectives et réécoutes critiques des performances passées jouent un rôle essentiel dans leur approche de l'improvisation et dans la construction du projet artistique qui la sous-tend.

15. Louis Armstrong était connu pour préparer ses solos. Comme le rappelle Brian Harker dans Louis Armstrong's Hot Five and Hot Seven Recordings, New York, Oxford University Press, 2011, «dans le jeu d'Armstrong, il semble qu'il y ait une différence qualitative entre les solos qui sont répétés (que ce soit sur scène ou en dehors de la scène) et ceux qui sont improvisés [...]. Nous n'avons pas de pistes alternatives pour «Oriental Strut», mais les notes péniblement répétées n'ont pas d'équivalent au sein des solos composés d'Armstrong. Il faut supposer que cette prise, comme beaucoup des premiers enregistrements des Hot Fives, correspond à une performance unique en studio. À l'inverse, la sûreté et la créativité dont Armstrong fait preuve dans le déploiement des arpèges de «Potato Head Blues» sembleraient indiquer qu'il y a eu, de la part d'Armstrong, un apprentissage délibéré, un processus d'expérimentation et de raffinement» (p. 86-87). Bien sûr, il s'agit là d'un cas limite : à bien des égards, on peut aussi considérer que de tels solos n'ont plus rien d'improvisé (ce que fait d'ailleurs Harker en distinguant les solos «improvisés » des solos «composés »)

16. Pour une description détaillée des séances de travail et autres « répétitions » de musiciens pratiquant l'improvisation libre, voir Canonne, «Rehearsing Free Improvisation?», art. cit. Plus généralement, il ressort également de ce travail que la question de la répétition n'est pas forcément taboue dans le monde des musiques librement improvisées, quand justement elle est envisagée comme faisant partie d'un travail «préparatoire » à la construction d'une identité de groupe. Comme le rappelle le pianiste Frédéric Blondy «un des trucs typiques de l'improvisateur qui vient de faire quelque chose, c'est qu'il fera rarement la même chose s'il doit faire une nouvelle improvisation. Par essence, il a envie... enfin, le musicien s'ennuie à répéter ce qu'il vient de faire. C'est un peu contradictoire avec l'idée de la musique improvisée, de refaire la même chose [...]. Mais en même temps, il faut accepter l'idée qu'il faut probablement qu'on passe par un temps où éventuellement on devrait faire la même musique, on devrait la répéter, la faire vivre, pour qu'on finisse par entendre tous le son du groupe » (Frédéric Blondy, en entretien avec Clément Canonne le 7 janvier 2016).

17. Voir Martin Guerpin, «Michael Brecker, le sportif et le virtuose», Les Cahiers du Jazz, nouvelle série, vol. 7, Paris, Outre Mesure, 2010, p. 47-58.

18. Gunther Schuller, Early Jazz: Its Roots and Musical Development, New York, Oxford University Press, 1968, p. x.

19. Ekkehard Jost, Free Jazz, New York, Da Capo Press, 1974

20. Ibid., p. 14.

21. Ibid. 
Si l'idée qu'il puisse exister des relations entre les improvisations d'un même musicien ou d'un même groupe peut apparaître comme une condition nécessaire au projet d'une génétique de l'improvisation musicale, la conception de l'improvisation comme work-in-progress permanent n'en soulève pas moins deux difficultés essentielles pour une telle approche. Celle, d'une part, des bornes du processus : peut-on le délimiter précisément, lui attribuer un début et une fin? Celle, d'autre part, de l'unité de ce processus. Peut-on identifier ce qui le sous-tend? Autrement dit, de quoi peut-on faire une étude génétique? L'examen de ces deux questions met en jeu la possibilité même de constituer un dossier de genèse, et plus généralement celle de transposer les outils de la critique génétique littéraire au domaine de l'improvisation musicale. Comment délimiter les portions de ce processus qui peuvent se prêter à une analyse génétique ? Quand y a-t-il un sens à considérer que deux improvisations peuvent être rassemblées au sein du même dossier génétique ? Répondre à ces questions implique d'envisager le transfert des concepts et des méthodes de la critique génétique littéraire au cas de l'improvisation musicale.

\section{De la génétique littéraire à la génétique de l'improvisation musicale : concepts, outils, méthodes}

\section{Délimiter le dossier de genèse d'une improvisation : le flou des frontières}

\section{LA DIFFICULTÉ D'IDENTIFIER UN TERMINUS AD QUEM}

La génétique littéraire s'attache à retracer la genèse d'un texte dont l'état final est relativement facile à identifier et qui constituera le terminus ad quem du dossier de genèse, son objet. Il s'agit le plus souvent d'une «œuvre» au sens de la version finale d'un texte éditée et publiée avec l'accord de l'écrivain. Dans cette version finale, le généticien peut «ne voir qu'un dérivé ultime des précédentes métamorphoses ou une entité extérieure à la sphère de l'avant-texte 22 ». On parlera ainsi du «dossier de genèse de Madame Bovary». Cette présentation succincte ne doit pas faire oublier des cas plus problématiques abordés par la génétique littéraire : celui par exemple des œuvres inédites et/ou laissées à l'état de brouillon ${ }^{23}$. Mais pour problématiques que soient ces cas, ce n'est rien au regard des difficultés soulevées par le domaine de l'improvisation musicale. Selon quels critères peut-on considérer une performance improvisée comme une version finale ou définitive dont il s'agira de retracer la genèse? Sauf exception, chaque improvisation peut en effet être envisagée comme une fin en soi. Comment identifier celle qui pourra être considérée comme un point remarquable?

Afin de ne pas définir arbitrairement et a posteriori une performance improvisée comme un terminus ad quem, il est possible de s'appuyer sur les gestes d'autorisation ou de publication des improvisateurs pour identifier les performances susceptibles de faire l'objet d'une enquête génétique. Ainsi, lorsqu'un improvisateur fait paraître un enregistrement d'une de ses performances (en concert ou en studio), il confère à cette dernière un statut particulier, puisque celle-ci fait alors l'objet d'un processus de sélection (le musicien devant la plupart du temps effectuer un choix, seul ou en concertation avec ses collègues et avec le producteur de la séance 24 , parmi d'autres improvisations enregistrées en studio ou captées en concert) la distinguant comme particulièrement réussie, ou du moins comme particulièrement représentative de sa production. Même s'il ne s'agit que d'un «document imparfait et incomplet d'une performance faisant partie d'une chaîne qui se construit tout au long de la carrière de l'improvisateur ${ }^{25}$ », il n'en demeure pas moins que c'est par cette performance, par cette improvisation, que le musicien a choisi de donner à entendre son travail. À ce titre, elle constitue un point remarquable au sein de la production du ou des musiciens considérés : non pas un «texte autonome ${ }^{26}$ » qui serait considéré pour

22. Pierre-Marc de Biasi, La Génétique des textes, Paris, Nathan, coll. «128», 2000, p. 70-71.

23. Claudine Gothot-Mersch, «L'édition génétique, le domaine français », dans Louis Hay (dir.), La Naissance du texte, Paris, Corti, 1989, p. 63-88. 24. Cette précision n'a rien d'anodin. Dans le monde du jazz, il arrive souvent que les musiciens qui n'enregistrent pas sous leur nom n'aient pas le dernier mot sur les prises choisies. Ainsi, si l'on s'intéresse à une improvisation de John Coltrane présente dans un disque de Miles Davis, il est possible que la version publiée par Miles Davis ne soit pas celle que John Coltrane aurait autorisée. Il arrive encore que le choix des prises publiées ne soit pas celui des musiciens, qui doivent parfois composer avec les préférences du directeur artistique et/ou du producteur de la séance d'enregistrement.

25. Peter Elsdon, Keith Jarrett's The Köln Concert, New York, Oxford University Press, 2013, p. 65. 
lui-même mais, au contraire, un terminus ad quem dont on pourrait tenter de retracer la genèse. L'argument de l'autorisation et de la stabilité que confère la publication d'une improvisation sous forme d'enregistrement peut aussi valoir pour d'autres formes de publicisation, moyennant quelques aménagements. C'est le cas du concert qui, en dehors des projets musicaux qui aboutissent à un enregistrement, intéresse au premier chef le généticien de l'improvisation.

Parallèlement à la diffusion par l'enregistrement, le fait même de se produire en concert constitue en effet une forme d'aboutissement et de publication. La performance d'une improvisation en live et en présence d'un public lui confère nécessairement un statut particulier par rapport aux improvisations que réalisent les musiciens lors de leurs séances de travail «privées ». Cet aspect est particulièrement significatif dans les pratiques d'improvisation libre : dans ce domaine, les concerts sont peu nombreux, ce qui leur confère un statut d'autant plus exceptionnel, comme le rappelle le saxophoniste Bertrand Denzler :

Il y a certains projets avec lesquels je travaille depuis longtemps et avec lesquels on a des périodes pendant lesquelles on travaille sans concert ou enregistrement à la clé, mais juste pour travailler. Mais au fond je suis assez convaincu que, jouer devant un public, c'est la vraie situation de l'improvisation... quand il y a une vraie présence physique d'un être humain écoutant dans la même pièce que moi, ça me met dans ce qu'on appelle le «temps réel». Cet auditeur représente le temps réel, je ne peux plus revenir en arrière, je ne peux pas passer cette étape [...]. Tant que je le ressentirai, je ferai des concerts 27 .

Dans un tel contexte, chaque improvisation en concert peut donc potentiellement devenir un point remarquable sur lequel s'arrêter, surtout quand on la rapporte aux improvisations «sans public» qui ont pu la précéder.

Même si toute improvisation peut être considérée par le généticien comme le terminus ad quem d'un dossier de genèse, les «points remarquables » identifiés dans le flux des performances d'un improvisateur sont donc principalement des performances choisies sur des critères d'autorisation ou de publication. Qu'elles soient publiées sur un disque ou encore jouées en concert, ce sont ces improvisations qui feront l'objet de l'analyse génétique, objet à partir duquel sera constitué le dossier de genèse.
Il convient ici de remarquer que ces performances « autorisées » ne sont pas forcément les manifestations les plus tardives (au sens chronologique) d'un processus. Il peut être très fécond de prendre en compte les improvisations ultérieures à celles identifiées comme des points remarquables : celles, par exemple, qui ont été enregistrées après la version d'un standard de jazz finalement retenue pour figurer sur un album. Mais ces prises ultérieures revêtent peut-être davantage d'intérêt dans le cadre d'une approche intertextuelle que dans le cadre d'une approche génétique. Elles peuvent par exemple aider l'analyste à identifier les propriétés communes essentielles à une série d'improvisations donnée. C'est ce que fait par exemple le musicologue Peter Elsdon dans son travail sur le Köln Concert (1975) de Keith Jarrett, en comparant cette célèbre performance improvisée avec d'autres improvisations réalisées avant ou après le concert de Cologne, mais durant la même tournée européenne. Elsdon met ainsi au jour les différents états musicaux et expressifs caractéristiques des improvisations en solo de Keith Jarrett pendant cette période, ainsi que la rhétorique guidant le passage d'un état à l'autre 28. Mais contrairement à l'approche d'Elsdon, la génétique implique de retracer la dynamique d'un processus créateur avec tout ce que cela suppose d'irréversibilité temporelle. Seules les performances précédant l'improvisation considérée (le «point remarquable») et appartenant au même projet peuvent donc prétendre faire partie du dossier génétique. Ce critère d'antériorité chronologique est essentiel. Il démarque notre approche génétique, qui tente essentiellement de rendre compte du passage d'une improvisation à une autre, de l'approche intertextuelle qui, elle s'intéresse plus généralement aux différents liens qu'une improvisation peut entretenir avec d'autres (qu'elles soient ou non du même musicien) ou avec des répertoires composés 29 .

Une fois élucidée la question du terminus ad quem, reste à déterminer ce qui constituera le point de départ, le terminus

\footnotetext{
26. Ibid.

27. Cité dans Canonne, «Rehearsing Free Improvisation?», art. cit. 28. Elsdon, op. cit., p. 43 et 45.

29. Il faudrait sans doute nuancer cette distinction; en particulier l'approche génétique a ceci de commun avec l'approche intertextuelle que toutes deux s'intéressent à des processus de redéploiement d'un texte à
} l'autre. 
a quo du dossier de genèse d'une improvisation considérée comme un point remarquable.

\section{LA DIFFICULTÉ D'IDENTIFIER UN TERMINUS A QUO}

D'après le second corollaire de Jost, selon lequel l'étude de l'improvisation d'un musicien nécessite de prendre en compte toutes les improvisations qu'il a déjà produites, le dossier génétique devrait donc se confondre avec l'ensemble de sa production antérieure. Cela reviendrait, dans le cas de la littérature, à inclure dans le dossier génétique d'une œuvre l'ensemble des textes produits par un écrivain depuis qu'il écrit. Or, il nous semble que Jost sous-estime tout ce que la carrière d'un improvisateur peut comporter de discontinuités. Certes, c'est bien le même musicien qui va improviser soir après soir, «développant un ensemble d'outils et de stratégies - non pas se répétant littéralement, mais travaillant avec les éléments d'un langage personnel ${ }^{30}$ » et ainsi forger et préciser son style d'improvisation. Mais ces improvisations se feront avec de nouveaux groupes, de nouveaux instruments, de nouvelles influences, de nouveaux cadres formels, et dans le cas du jazz, à partir de nouvelles compositions ou de nouveaux arrangements. Autant de changements qui exercent le plus souvent une influence considérable sur les performances d'un improvisateur donné. Il n'y a ainsi pas grand sens à vouloir examiner les courtes improvisations qu'affectionne Keith Jarrett en concert solo depuis le début des années 2000 à l'aune des grandes fresques qui caractérisaient sa pratique dans les années 1970, tant les enjeux et les modalités de ces performances sont distincts. De même, quand Howard Brofsky 31 entreprend de retracer, conformément à l'idée de l'improvisation comme work-in-progress, la progression des solos de Miles Davis sur «My Funny Valentine», des esquisses de 1956 et 1958 , avec leurs moments uniques jusqu'à «la conception globale de la performance de 1964 », cela ne peut se faire qu'en passant sous silence tous les éléments cruciaux qui rendent ces différentes versions largement étrangères les unes aux autres, et au premier chef le fait que ces solos sont construits en interaction avec des sections rythmiques aux orientations stylistiques et aux comportements radicalement différents.

Nous proposons donc, dans le cas du jazz, d'envisager comme terminus a quo d'un dossier génétique la première trace d'un travail ou d'une performance improvisée sur un morceau donné, par un musicien évoluant au sein d'une formation stable. On pourra par exemple étudier la genèse de l'improvisation de Charlie Parker sur «Billie's Bounce», enregistrée le 26 novembre 1945 et publiée la même année par le label Savoy 32 . Outre cette version publiée, le dossier comprendra les trois premiers enregistrements (appelés prises) réalisés lors de la même séance, et qui ont pu faire l'objet de réinvestissements successifs. Le cas de l'improvisation libre est différent car cette pratique se déploie généralement sans référent, ou à partir de consignes minimales. Le terminus a quo peut alors correspondre à la première improvisation effectuée par un groupe donné d'improvisateurs, et donc au moment où commence à se forger l'identité artistique du groupe en question. On pourra par exemple étudier, à partir des archives personnelles des musiciens, la manière dont les textures collectives expérimentées par le quartet franco-norvégien Dans les arbres lors de leurs premières séances de travail commun en juillet 2006 ont pu se retrouver mobilisées au sein des improvisations enregistrées quelques jours plus tard pour le label ECM.

Il ressort de l'examen de ces différentes difficultés que le projet d'une génétique de l'improvisation ne peut aboutir qu'en rejetant les deux conceptions les plus communément défendues de l'improvisation (d'un côté, la conception de l'improvisation comme pure singularité, sans passé ni avenir; de l'autre, la conception de l'improvisation comme perpétuel work-in-progress) au profit d'une conception hybride, admettant à la fois l'existence de continuités pouvant lier entre elles un certain nombre d'improvisations successives (à la fois par le souvenir des performances passées et par l'anticipation de performances à venir) et l'existence de discontinuités permettant de distinguer différentes phases tout au long de la carrière d'un improvisateur (ou d'un groupe d'improvisateurs). Chaque dossier génétique permettra de documenter une de ces phases, chacune identifiée par une configuration commune : stabilité du contexte de performance, stabilité du personnel

30. Elsdon, op. cit., p. 65.

31. Howard Brofsky, «Miles Davis and My Funny Valentine: The Evolution of a Solo », Black Music Research Journal, vol. 3, 1983, p. 23-45.

32. Charlie Parker All Stars, Billie's Bounce/Now's The Time, enregistré à New York le 26 novembre 1945, Savoy 573. 
improvisant, stabilité de l'instrumentarium utilisé et, le cas échéant, stabilité du matériau compositionnel - thème, grille harmonique, structure, arrangement, etc. - auquel s'articule l'improvisation. Bien entendu, il y a forcément une grande part de subjectivité, voire d'arbitraire, dans la délimitation précise de ces «projets» comme dans le fait de décider d'y associer ou non telle ou telle improvisation. Mais comme le rappelle Daniel Ferrer, le fait même de rattacher un ensemble de manuscrits à une œuvre achevée ou même de relier certains manuscrits entre eux ne va pas non plus de soi 33 . Il y a là quelque chose d'inhérent à l'entreprise génétique, qui présuppose toujours un ensemble de décisions critiques de la part de l'analyste.

Il n'en reste pas moins vrai que c'est dans l'acte de délimitation d'un dossier de genèse d'une improvisation considérée comme un point remarquable que la génétique de l'improvisation se distingue d'une stylistique de l'improvisateur, comprise comme mise en évidence du vocabulaire instrumental et des stratégies discursives caractéristiques développées par un improvisateur tout au long de sa carrière ${ }^{34}$. Alors que l'approche stylistique est peut-être condamnée à prendre en compte l'intégralité de la production d'un improvisateur (encore que ce point soit sans doute également contestable), la génétique de l'improvisation s'attache à reconstituer la genèse d'une performance improvisée particulière. Elle peut s'appuyer sur les continuités qui unissent certaines des performances d'un improvisateur comme sur les discontinuités qui ponctuent son travail pour délimiter l'ensemble des performances relevant d'un même projet improvisatoire. L'objet d'une génétique de l'improvisation est précisément ce projet, ainsi que ses différentes réalisations concrètes («instanciations ») et toutes les opérations observables de l'une à l'autre de ces instanciations.

\section{La notion de projet improvisatoire et l'objet de la génétique de l'improvisation}

La génétique de l'improvisation musicale suppose en effet l'existence de projets improvisatoires communs à plusieurs improvisations, définis comme des ensembles de propriétés (plus ou moins vagues) instanciés par une séquence donnée d'improvisations. La notion de projet occupe une place importante dans la génétique littéraire : c'est elle en effet qui sous-tend le passage d'une version préparatoire à l'autre d'un texte :

Une très grande part de ce qui est développé dans le brouillon est déjà en germe dans le scénario. On peut considérer plus généralement que toute nouvelle version est en partie incluse dans la première - mais la difficulté est de déterminer quelle partie 35 .

L'analyste peut alors croiser différents critères heuristiques pour mettre au jour la persistance d'un même projet au sein d'un ensemble d'improvisations par-delà la singularité et la variété de chaque improvisation considérée individuellement, qu'il s'agisse des critères portant sur les conditions de l'improvisation ou de critères portant sur la description de la musique effectivement improvisée (stabilité du matériau instrumental, stabilité des stratégies d'interaction entre les musiciens, stabilité dans la conduite globale de la performance, etc.). L'un des objectifs de la génétique de l'improvisation consiste donc à identifier un projet improvisatoire sous-tendant un ensemble donné de performances, regroupé au sein d'un dossier de genèse. Et lorsque l'existence de ce projet est avérée, la tâche du généticien consiste à l'élucider et à décrire son incidence sur les opérations observables d'une version à l'autre des improvisations regroupées dans un même dossier.

Dans le cas du jazz, un projet peut ainsi être défini comme la lecture particulière qu'offre un musicien (ou un groupe de musiciens) d'un standard ou d'une composition originale : l'ensemble des choix interprétatifs (au sens large) qui caractérisent sa performance, sa manière de jouer (et d'improviser sur) telle ou telle composition à un certain moment de sa carrière. À la manière d'un interprète de musique classique qui effectue un certain nombre de choix face à la partition qu'il se propose d'exécuter choix qu'il pourra ensuite reconduire d'une performance

33. Voir Daniel Ferrer, Logiques du brouillon, Paris, Le Seuil, 2011, p. 100. 34. Pour un exemple de ce type d'analyse, voir Thomas Owens, Charlie Parker. Techniques of Improvisation, PhD diss., University of California, Los Angeles, 1974, 2 vol. et Thomas Owens, Bebop. The Music and its Players, New York, Oxford University Press, 1995, p. 28-45.

35. Daniel Ferrer, «L'écriture et l'accident», dans Olga Anokhina et Sabine Pétillon (dir.), Critique génétique. Concepts, méthodes, outils, Saint-Germain-la-Blanche-Herbe, Imec éditeur, coll. «Inventaires », 2009 , p. 125 . 
à l'autre ${ }^{36}$ - les musiciens de jazz sont traditionnellement amenés à spécifier davantage le canevas (la «grille») qui sert typiquement de support à leurs improvisations. C'est cette opération de spécification que l'on peut par exemple observer dans le cinquième volume de la «Bootleg Series» que le label Columbia consacre au trompettiste Miles Davis, qui comprend notamment toute la répétition ayant précédé l'enregistrement de la première prise du Freedom Jazz Dance d'Eddie Harris (publié initialement en 1966 sur l'album Miles Smiles) et au cours de laquelle on entend les musiciens du deuxième quintet mettre progressivement au point leur propre version de ce thème, par une alternance d'indications verbales du trompettiste, de prises d'initiatives individuelles et de courtes discussions collectives ${ }^{37}$.

En revanche, l'idée même d'un projet commun à plusieurs improvisations peut sembler particulièrement contre-intuitive dans le cas de l'improvisation libre. Mais l'observation ethnographique de la pratique de groupes d'improvisation libre a pu montrer que les musiciens n'avaient la plupart du temps guère de peine à définir leur identité de groupe et, partant, à énoncer les caractéristiques principales communes à leurs performances improvisées 38 . Ces caractéristiques résultent parfois de choix délibérés effectués en amont ou au début du travail en commun 39 mais, le plus souvent, elles sont le fruit d'une sédimentation des pratiques individuelles et collectives au sein du groupe :

Dans les groupes d'improvisation dans lesquels je suis et qui durent longtemps, ça s'est toujours passé comme ça. Au bout d'un moment, le territoire et le son du groupe, ce que j'appelle l'espace musical du groupe, devient une sorte d'évidence, c'est un monde, un univers en soi. On peut le tordre, on peut jouer avec ses limites, on peut essayer de le détruire, de le reconstruire, on peut essayer de le changer mais je pense qu'il est presque indestructible, à la fois humainement et musicalement 40 .

Reconnaitre l'existence de tels projets improvisatoires apparait bien comme une condition nécessaire pour entreprendre une génétique de l'improvisation musicale. Elle permet en effet de répondre à l'exigence de réidentification et de continuité que doivent satisfaire les produits artistiques qu'envisage la génétique : sans critère nous permettant d'établir que, en un sens, c'est bien le même projet qui se poursuit d'une improvisation à l'autre (et donc que c'est bien le même projet que l'on peut réidentifier à travers des performances qui présentent toutes des singularités irréductibles) il ne peut y avoir de génétique de l'improvisation.

En termes ontologiques, ces projets s'apparentent donc à des types plus ou moins «épais», pour reprendre le vocabulaire de Stephen Davies41, types dont les improvisations en question seraient les instances ${ }^{42}$. Cela pose un problème considérable dans le cadre d'une génétique de

36. Ainsi, Glenn Gould nous a laissé deux «lectures » des Variations Goldberg de Bach, celle de 1955 et celle de 1981. S'il n'y a jamais eu d'exécution de la lecture de 1981 puisque Gould s'était retiré de la scène depuis de nombreuses années déjà, la lecture de 1955 a en revanche donné lieu à de multiples exécutions en concert dans les années qui ont suivi cet enregistrement, et il subsiste au moins un témoignage enregistré (enregistrement live à Salzbourg de 1959) nous permettant de dire qu'il s'agissait là d'exécutions de la même lecture (identité des choix interprétatifs pour l'ornementation, les tempi, les reprises, etc.).

37. Notons que faire la génétique de cette lecture est un projet bien distinct de celui d'une génétique des improvisations réalisées par les musiciens sur ce Freedom Jazz Dance. D'ailleurs, il est remarquable de constater qu'il n'y a pas d'improvisation à proprement parler tout au long de ces 23 minutes, les musiciens s'interrompant toujours au seuil du premier chorus, réservant en quelque sorte leurs improvisations pour la première «vraie» prise. Si un tel enregistrement permet donc de retracer la genèse d'un arrangement «sur le vif», il ne nous permet pas vraiment d'éclairer les improvisations de la master take qui lui succède.

38. Voir Canonne, «Rehearsing Free Improvisation?», art. cit.

39. Le batteur Antonin Gerbal indique ainsi que, dans le cas du trio Zoor, les musiciens ont défini un certain nombre de préalables : «Parfois, je fonctionne plus par axiomes. Si je te dis : une forme musicale, qui va d'un point $\mathrm{A}$ à un point $\mathrm{B}$, avec un développement, bon bah tu peux déjà te faire une idée de l'improvisation que ça va donner. On a aussi décidé de jouer au chrono, comme ça on ne se pose plus la question de la durée. C'est que des blocs de 40 minutes, des tranches de temps... on parlait aussi beaucoup de pulsation très lente, au début, d'une pulsation infiniment lente... et d'un son... déjà, d'utiliser nos instruments en évitant les techniques étendues... habiter une pulsation lente, dans une tranche de temps objective» (Antonin Gerbal, en entretien avec Clément Canonne le 18 février 2016).

40. Bertrand Denzler, en entretien avec Clément Canonne le 18 novembre 2015.

41. Voir Stephen Davies, Musical Works and Performances: A Philosophical Exploration, New York, Oxford University Press, 2001. Un type «fin» (par exemple la grille harmonique de My Funny Valentine) détermine moins précisément le contenu de ses instances que ne le fait un type «épais» (par exemple la Deuxième Sonate pour piano de Pierre Boulez). Dans cette perspective, on peut considérer que la lecture proposée par Miles Davis et les musiciens du deuxième quintet de My Funny Valentine est un type beaucoup plus épais que le projet musical sous-jacent aux différentes improvisations du trio The Necks, dans la mesure où il détermine beaucoup plus précisément le contenu des performances qui l'instancient. 
l'improvisation musicale : si les improvisations sont les instances d'un type, alors leur genèse s'explique entièrement par la relation d'instanciation. Si nous souhaitons comprendre la manière dont le pianiste Mikhaïl Pletnev exécute les Tableaux d'une exposition de Moussorgsky à tel ou tel moment de sa carrière, la mise au jour de sa lecture particulière de l'œuvre, progressivement élaborée lors de séances de travail autour de la partition, s'avérera bien plus utile qu'une comparaison de cette exécution avec ses interprétations précédentes de l'œuvre. Dans cette même perspective, l'improvisation $n$ est ce qu'elle est en vertu du projet improvisatoire qu'elle instancie, et non en raison d'une relation particulière qui l'unirait à l'improvisation $n-1$ qui la précède. Bien sûr, au moment de jouer l'improvisation $n$, les improvisateurs peuvent avoir présent à l'esprit l'improvisation $n-1$ qu'ils viennent juste de réaliser, mais la relation intentionnelle qui unit les deux performances sera bien moins déterminante pour rendre compte de l'improvisation $n$ que la relation d'instanciation qui unit l'improvisation $n$ au projet improvisatoire commun aux improvisations $n$ et $n-1$.

De tels «projets improvisatoires » n'ont certes pas d'existence indépendante des performances qui les instancient : d'une part, ils émergent (plus ou moins progressivement) des performances des musiciens elles-mêmes - devenant plus précis ou plus explicites au fur et à mesure que les improvisations s'enchaînent; d'autre part, ils ne sont évidemment pas immuables et chaque performance peut théoriquement contribuer à les redéfinir. Cependant, une fois sédimentés et incorporés par les musiciens, on peut supposer qu'ils ne se modifient en réalité que marginalement, jusqu'à ce qu'ils soient effectivement abandonnés au profit d'un nouveau projet 43 . Il y a donc bel et bien un sens à dire que les musiciens du trio The Necks instancient leur projet improvisatoire (une fois celui-ci relativement stabilisé, quand bien même ses contours en resteraient quelque peu fluctuants) à chacune de leurs performances, de la même manière que Pletnev instancie sa lecture des Tableaux (une fois que celle-ci s'est pleinement cristallisée) à chacune de ses performances de cette œuvre.

Il nous semble donc nécessaire d'ajouter un ultime critère pour donner un sens à l'entreprise d'une génétique de l'improvisation musicale - à savoir un critère de précocité temporelle. Plus l'on considère un projet improvisatoire dans ses manifestations tardives, plus il est sédimenté, plus la relation d'instanciation joue un rôle déterminant dans l'explication de la performance, au détriment de la relation intentionnelle pouvant exister entre cette performance et une ou plusieurs performances antérieures. Mais à l'inverse, plus on considère un projet improvisatoire dans ses premières manifestations, plus la relation de dérivation intentionnelle - le fait que les musiciens improvisent «à partir» d'une performance précédente (que ce soit parce que cette performance vient d'avoir lieu, ou parce qu'elle vient de faire l'objet d'une réécoute critique) - peut jouer un rôle clé dans la restitution du processus génétique considéré (voir fig. 2 pour un schéma clarifiant la dynamique des projets improvisatoires).

Plus encore : en s'intéressant aux premières manifestations d'un projet improvisatoire, on peut également mettre au jour la genèse du projet lui-même. Celui-ci ne préexiste pas entièrement aux performances qui l'instancient; il est également engendré par ces mêmes performances. L'application de ce critère de précocité temporelle parachève le modèle que nous proposons pour une génétique de l'improvisation musicale. Celle-ci combine donc une génétique de la performance improvisée, qui met en lumière les opérations de dérivation intentionnelle permettant de rendre compte du passage d'une improvisation à une autre, et une génétique du projet improvisatoire - qui montre comment le projet émerge précisément à partir de la concaténation de ces relations de dérivation intentionnelle. De même que l'écriture, qui n'est pas une pure transposition du projet de l'écrivain et peut contribuer, par le jeu des «accidents » endogènes, à le modifier ${ }^{44}$, la génétique de l'improvisation se donne pour objet l'élucidation de la relation dialectique à l'œuvre entre les modifications apportées à chaque nouvelle instanciation du projet et celles que ces instanciations elles-mêmes font subir à ce projet.

Après avoir élucidé ces questions théoriques et précisé l'objet de la génétique, il reste à aborder de manière concrète la constitution d'un dossier de genèse. Peut-on identifier,

42. Pour une présentation du couple conceptuel type/instance dans le domaine de l'ontologie de la musique, voir Clément Canonne et Pierre Saint-Germier, «Les œuvres de musique et leur ontologie» dans Jerrold Levinson, Essais de philosophie de la musique, Paris, Vrin, 2015, p. 53-73.

43. Les témoignages de musiciens discutés dans l'article «Rehearsing Free Improvisation?», op. cit., semblent du moins aller en ce sens. 44. Daniel Ferrer, «L'écriture et l'accident», op. cit., p. 124-125. 


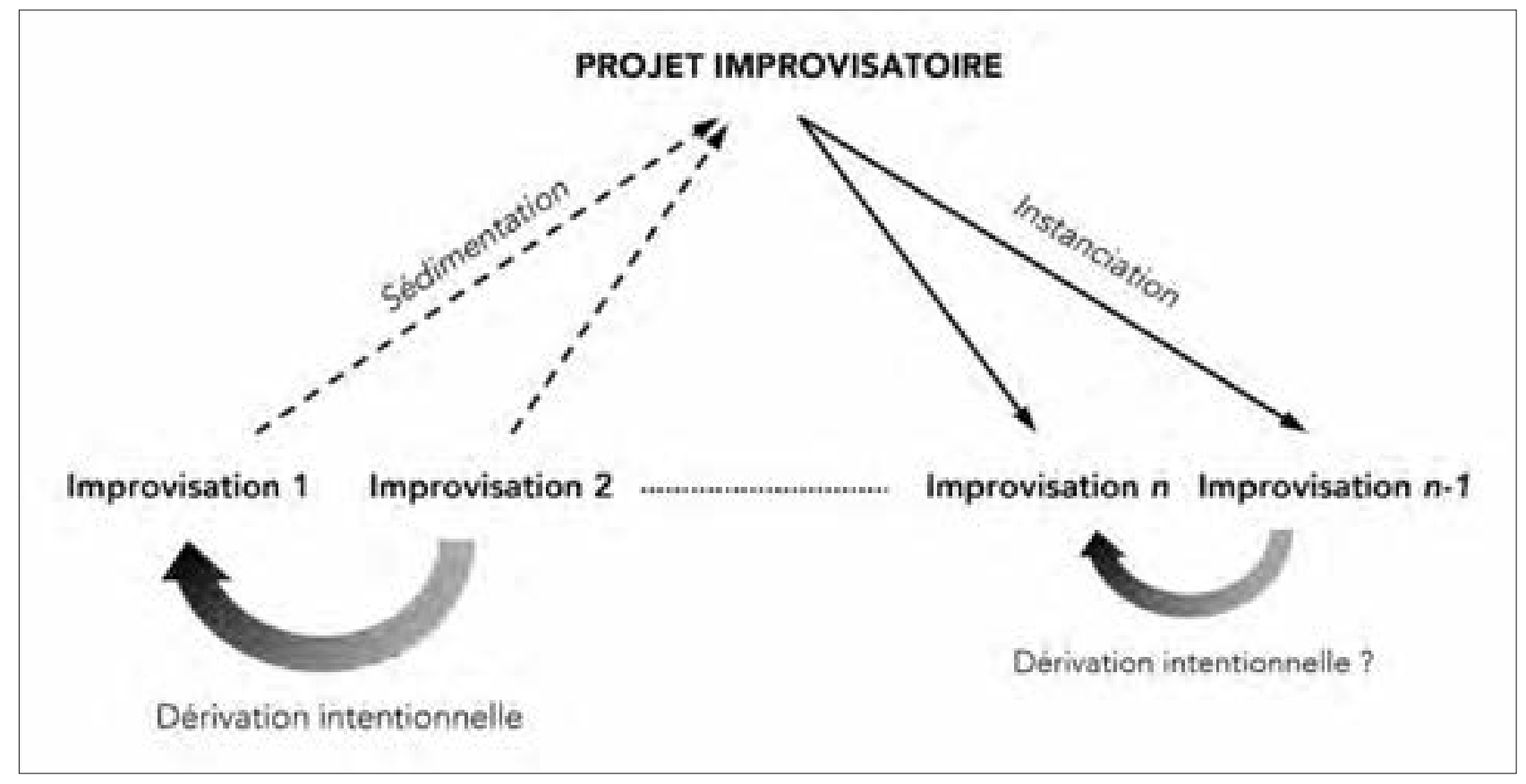

Fig. 2 : La relation génétique, une dialectique entre instanciation et dérivation intentionnelle.

dans le cas de l'improvisation, des documents dont le statut pourrait être l'équivalent de celui des «avant-textes» et des «brouillons» littéraires? Voilà la question que nous traiterons dans la seconde livraison de cet article, avant de proposer une typologie des approches génétiques de l'improvisation musicale.

À suivre...

MARTIN GUERPIN est agrégé de musique, lauréat du prix Socan-Proctor (2013) et du prix de la Société québécoise de recherche en musicologie (2013). Il prépare un doctorat sur les «Enjeux esthétiques de la réception du jazz dans le monde musical savant parisien de l'entre-deux-guerres ». Ses recherches portent également sur les processus de création à l'œuvre dans l'improvisation. Ses travaux ont fait l'objet de publications dans les Cahiers de la SQRM (2014) et dans Eurojazzland. Il enseigne au Conservatoire de Paris et à l'Institut d'études politiques de Paris. Depuis 2014, il fait partie du comité directeur des Cahiers du Jazz. Saxophoniste, il se produit régulièrement avec le Gil Evans Paris Workshop de Laurent Cugny.

martin.guerpin@gmail.com

CléMENT CANONNE est chargé de recherche au CNRS, rattaché à l'équipe Analyse des pratiques musicales au sein de l'UMR 9912 «Sciences et technologies de la musique et du son» (IRCAM-CNRS-Sorbonne Université). Ses recherches portent principalement sur la question de l'improvisation, envisagée à la fois comme pratique et comme paradigme. Son travail récent a fait l'objet de publications dans plusieurs revues internationales (Cognition, Music Theory Online, Revue de musicologie, Psychology of Music, etc.). Il s'intéresse également à la philosophie de la musique, a dirigé un ouvrage collectif : Perspectives philosophiques sur les musiques actuelles (Delatour, 2017) et a traduit et introduit, en collaboration avec Pierre Saint-Germier, une sélection des Essais de philosophie de la musique de Jerrold Levinson (Vrin, 2015).

clementcanonne@hotmail.com 


\section{Pour une génétique de l'improvisation musicale (première partie)}

Le projet même d'une génétique de l'improvisation musicale peut sembler largement paradoxal : d'un côté, chaque improvisation se présente à nous comme une pure singularité, une performance musicale éphémère sans passé ni avenir; d'un autre côté, chaque improvisation porte en elle la trace de processus de mémorisation, d'incorporation et de réinvention qui sont consubstantiels à la pratique des improvisateurs et qui se déploient d'une performance à l'autre, fondant ainsi tout acte d'improvisation en une sorte de work-inprogress permanent. La première partie de notre article se propose donc d'identifier les conditions de possibilité d'une génétique de l'improvisation musicale dans le champ du jazz et des musiques librement improvisées. Ce faisant, nous introduisons la notion de «projet improvisatoire» et proposons un modèle dynamique de la relation génétique à l'œuvre dans le travail des improvisateurs.

The project of a Genetics of musical improvisation can in itself seem quite paradoxical. On the one hand each improvisation appears to us as a pure singularity, an ephemeral musical performance without past or future, and on the other hand, each improvisation bears the trace of a process of memorization, incorporation and reinvention that are consubstantial with improvisers' practice and that unfold from one performance to another, thus making any act of improvisation a sort of permanent work in progress. We will first identify the conditions for the possibility of a Genetics of musical improvisation in Jazz and other types of freely improvised music. In doing so we will introduce the notion of "improvisational project" and suggest a dynamic model of the genetic relationship at play in improvisers' work.

Schon das Projekt einer génétique der musikalischen Improvisation mag weitgehend paradox erscheinen: Einerseits präsentiert sich jede Improvisation als reine Singularität, als ephemere musikalische Leistung ohne Vergangenheit oder Zukunft. Andererseits trägt jede Improvisation die Spur von Prozessen des Memorierens, des Inkorporierens und des Neuerfindens, die der Praxis der Improvisatoren wesenseigen sind und die sich von einer Aufführung zur anderen entfalten. Somit gründet jeder Akt von Improvisation in einer Art des permanenten work in progress. Der erste Teil unseres Artikels beabsichtigt die Bedingungen der Möglichkeit einer génétique der musikalischen Improvisation im Bereich des Jazz und der frei improvisierten Musik auszumachen. Dabei führen wir den Begriff ,projet improvisatoire“ ein und stellen ein dynamisches Modell der genetischen Beziehung zum Werk in der Arbeit der Improvisatoren vor.
El proyecto mismo de una genética de la improvisación musical puede parecer muy paradójico: por un lado, cada improvisación se nos presenta como una pura singularidad, una experiencia musical efímera sin pasado ni futuro; por el otro, cada improvisación lleva en sí misma las huellas del proceso de memorización, reincorporación y reinvención que son consubstanciales a la práctica de los improvisadores y que se despliegan en los distintos desempeños, convirtiendo cada acto de improvisación en una suerte de work in progress permanente. La primera parte de nuestro artículo se propone identificar las condiciones de posibilidad de una genética de la improvisación musical en el campo del jazz y de las músicas libremente improvisadas. Con ello, introducimos la noción de "proyecto improvisatorio" y proponemos un modelo dinámico de la relación genética que interviene en el trabajo de los improvisadores

Falar de genética da improvisação musical pode parecer bastante paradoxal como projeto: por um lado, cada improvisação se apresenta como singularidade pura, como efémero ato musical sem passado nem futuro; por outro lado, cada improvisação traz consigo vestígios de processos de memorização, incorporação e reinvenção que estão enraizados na prática dos improvisadores e que se desdobram de um para outro ato, assim convertendo a improvisação numa espécie de permanente work in progress. Neste artigo explora-se a viabilidade duma genética da improvisação musical no campo do jazz e do improviso livre. Para tal, é introduzido o conceito de 'projecto improvisatório' e proposto um modelo dinâmico para a relação genética que opera no trabalho dos improvisadores.

L'idea stessa di una genetica dell'improvvisazione musicale può sembrare decisamente paradossale: ogni improvvisazione si presenta come una pura singolarità, una performance musicale effimera, senza passato né avvenire. D'altra parte, ogni improvvisazione reca le tracce dei processi di memorizzazione, incorporazione e reinvenzione consustanziali alla pratica degli improvvisatori, che si dispiegano da una performance all'altra, fondando così ogni atto d'improvvisazione come una sorta di permanente work in progress. La prima parte del nostro articolo si propone d'identificare le condizioni di possibilità di una genetica dell'improvvisazione musicale nel campo del jazz e delle musiche liberamente improvvisate. S'introduce in seguito la nozione di "progetto improvvisatorio" e si propone un modello dinamico della relazione genetica che interviene nel lavoro degli improvvisatori. 